\title{
Disulfiram's Antineoplastic Effects on Ovarian Cancer
}

\author{
Youssef A. Rezk1, Kun Yang2, Shoumei Bai'2, Karen Mclean1, Carolyn Johnston1, \\ R. Kevin Reynolds ${ }^{1}$, Ronald J. Buckanovich ${ }^{1,2 *}$ \\ ${ }^{1}$ Division of Gynecologic Oncology, Department of Obstetrics and Gynecology, University of Michigan, \\ Ann Arbor, MI, USA \\ ${ }^{2}$ Division of Hematology Oncology, Department of Internal Medicine, University of Michigan, \\ Ann Arbor, MI, USA \\ Email: "ronaldbu@umich.edu
}

Received 22 October 2015; accepted 8 December 2015; published 11 December 2015

Copyright (C) 2015 by authors and Scientific Research Publishing Inc.

This work is licensed under the Creative Commons Attribution International License (CC BY).

http://creativecommons.org/licenses/by/4.0/

(c) (†) Open Access

\section{Abstract}

Objective: Aldehyde dehydrogenase (ALDH) enzymatic activity identifies ovarian cancer stem-like cells. We investigated the antineoplastic activity of the ALDH inhibitor Disulfiram on bulk ovarian cancer cells and $\mathrm{CD}_{133}{ }^{+} / \mathrm{ALDH}^{+}$cancer stem-like cells. Study Design: Ovarian cancer cell lines, human ovarian surface epithelial cells, and mesenchymal stem cells were treated with increasing concentrations of Disulfiram and/or Cisplatin in vitro. Treated cells were assessed for viability or FACS-analyzed for either percentage of ovarian cancer stem-like cells or induction of apoptosis. Disulfiram's impact on cancer stem-like cells was tested in vitro using tumor sphere formation assays and in vivo using tumor initiation assays with in vitro-treated A2780 cells in NSG mice. Finally, Disulfiram's in vivo activity was assessed versus $\mathrm{CD}^{2} 3^{+} / \mathrm{ALDH}^{+}$cell-initiated tumor xenografts. Results: Disulfiram demonstrated antineoplastic activity against multiple ovarian cancer cell lines. While Disulfiram had limited in vitro toxicity against human ovarian surface epithelial cells or mesenchymal stem cells (IC50 of $\sim 15 \mu \mathrm{M}$ and $>30 \mu \mathrm{M}$, respectively), its antineoplastic activity against cell lines was comparable to Cisplatin $\left(\mathrm{IC}_{50} \sim 1.5 \mu \mathrm{M}\right)$. Disulfiram-mediated cell death was due, at least in part, to induction of apoptosis. Disulfiram activity was additive with chemotherapy. Disulfiram demonstrated selective depletion of $\mathrm{CD}_{4} 4^{+}$cells but not the $\mathrm{CD} 133^{+}$cancer stem-like cells. Disulfiram had no therapeutic impact on tumor initiation studies or in vivo therapy of whole cell line or stem cell-initiated tumor xenografts. Conclusions: In biologically relevant concentrations, Disulfiram has clear antineoplastic activity against ovarian cancer cells in vitro. Disulfiram selectively depleted $\mathrm{CD44}^{+}$but not $\mathrm{CD} 133^{+}$ovarian cancer stem-like cells in vitro. However, Disulfiram had no significant activity in vivo. Thus, improved and more selective ALDH inhibitors may be required to target ovarian cancer stem cells.

\footnotetext{
*Corresponding author.
}

How to cite this paper: Rezk, Y.A., Yang, K., Bai, S., Mclean, K., Johnston, C., Reynolds, R.K. and Buckanovich, R.J. (2015) Disulfiram's Antineoplastic Effects on Ovarian Cancer. Journal of Cancer Therapy, 6, 1196-1205. 


\section{Keywords}

\section{Antineoplastic, ALDH, Disulfiram, NSG Mice, Ovarian Cancer Stem Cells}

\section{Introduction}

Many solid tumors contain a population of cells, termed cancer stem-like cells (CSC). CSC, despite constituting a small percentage of the overall cancer cell population, have increased tumor-initiation capacity and have therefore been proposed as a source of disease recurrence [1]. As such, CSC have been proposed as a therapeutic target. Aldehyde dehydrogenase enzymatic activity (ALDH) has been identified as a marker of CSC in numerous malignancies, including ovarian cancer [2] [3]. ALDH-high $\left(\mathrm{ALDH}^{+}\right)$epithelial ovarian cancer cells are chemotherapy-resistant; can both initiate and propagate tumors in mice, and can create heterogeneous lineages of ovarian cancer cells [3]. Thus, they meet the American Association for Cancer Research consensus criteria for CSC [4]. Indeed, we have shown that ALDH and CD133 identify an ovarian cancer differentiation hierarchy [5].

There is additional evidence that $\mathrm{ALDH}^{+}$cells are critical in ovarian cancer: 1) the presence of cells positive for ALDH1A1 and prominin-1 (CD133, a CSC marker) in patients' primary tumor specimens correlates with poor outcome [3]; 2) ALDH1A1 is upregulated 100-fold in ovarian cancer cells selected for resistance to either Cisplatin or Paclitaxel; 3) siRNA knockdown of ALDH1A1 restores chemosensitivity [6]; and 4) ALDH1A1 and CD133 are enriched in tumor specimens immediately following chemotherapy [7]. Based on the preferential activity of ALDH in chemotherapy-resistant tumor-propagating cells, ALDH has been implicated as a therapeutic target.

ALDH enzymes catalyze the oxidation of aldehydes to their carboxylic acid forms. These enzymes play an important role in various cellular metabolic pathways including the detoxification of acetaldehyde, the detoxification of free oxygen radicals generated by chemotherapeutic agents (such free oxygen radicals can lead to cancer cell membrane peroxidation and subsequent apoptosis), and the conversion of retinal to retinoic acid to regulate cellular transcription and differentiation pathways [8].

Disulfiram is an orally available ALDH inhibitor which has been used for decades in the treatment of chronic alcoholism. While Disulfiram was primarily developed to inhibit ALDH2, Disulfiram also inhibits ALDH1 isozymes. Based upon its ability to inhibit ALDH1 isozymes, Disulfiram has recently been tested as a CSC-targeting drug. Disulfiram has been reported to have anti-cancer activity in numerous malignancies, including breast, colon and prostate cancers among others [9]-[14]. Studies in breast cancer have suggested that Disulfiram may specifically target the breast CSC population [11]. More recently, it was shown that ALDH activity was necessary to maintain a drug-resistant phenotype in cancer cells and that ALDH inhibition with Disulfiram, via modulation of reactive oxygen species, could overcome this drug-resistant phenotype and delay disease recurrences [15].

Given that we and others have defined ALDH as a marker of ovarian CSC, and given the potential of Disulfiram to target $\mathrm{ALDH}^{+} \mathrm{CSC}$, we tested the ability of Disulfiram to target ovarian CSC both in vitro and in vivo in Nod-Scid-Gamma (NSG) mice.

\section{Materials and Methods}

\subsection{Cell Lines and Cytotoxicity Assays}

A2780, OVCAR3, OVCAR8, HEY1, and OVSAHO cells were grown in RPMI media (Thermo Scientific, Rockford, IL) while SKOV3 and human ovarian surface epithelial (HOSE) cells were maintained in DMEM media (Gibco, Grand Island, NY) supplemented with 10\% fetal bovine serum (Invitrogen, Carlsbad, CA) and $1 \%$ penicillin/streptomycin (Invitrogen) at $37^{\circ} \mathrm{C}$ with $5 \% \mathrm{CO}_{2}$. Mesenchymal stem cells (MSC, purchased from Invitrogen) were grown in serum-free MEBM-2 media (Lonza, Walkersville, MD) supplemented with 2\% B27 (Gibco), EGF (1:5000 dilution, Invitrogen), 1\% penicillin/streptomycin (Invitrogen), hydrocortisone (1:50,000 dilution, Sigma, St. Louis, MO), insulin (1:800 dilution, Invitrogen), BME (1:125 dilution, Sigma), and 0.5\% bFGF (BD Biosciences, San Jose, CA). Cell lines were seeded onto 96-well plates at a density of 7500 cells/well. The following day, cells were treated with increasing concentrations (range $0.3-30 \mu \mathrm{M}$ ) of Disulfiram or Cis- 
platin for 3 days; then cell viability was assessed using the methylthiazoltetrazolium (MTT) assay as previously described [16].

\subsection{Tumor Sphere Assays}

SKOV3 cells, primary patient ascites, or FACS sorted primary cells were plated in triplicate (2000 cells/well for SKOV3 and 10,000 cells/well for patient samples) on ultra-low attachment plates (Corning, Tewksbury, MA) in serum-free MEBM-2 media (Lonza) supplemented with 2\% B27 (Gibco), EGF (1:5000 dilution, Invitrogen), 1\% penicillin/streptomycin (Invitrogen), hydrocortisone (1:50,000 dilution, Sigma), insulin (1:800 dilution, Invitrogen), BME (1:125 dilution, Sigma), and 0.5\% bFGF (BD Biosciences). Cells were treated with the indicated concentrations of Disulfiram for 3 days. Sphere formation was assessed on day 15 (SKOV3) or 20 (patient samples); triplicate wells were photographed using the Olympus Microsuite Biological Suite software and total sphere number per well was counted. Results were compared using Student t-test.

\subsection{In Vivo Studies}

Nude mice were obtained from Charles River Laboratories. Animals were maintained in accordance with institutional policies, and all studies were performed with approval of the University Committee on Use and Care of Animals (UCUCA) of the University of Michigan. To determine the tumor-initiating capacity of in vitro-treated bulk ovarian cancer cells, A2780 cells were treated with Disulfiram $(2 \mu \mathrm{M}$ and $10 \mu \mathrm{M})$ or Cisplatin $(0.25 \mu \mathrm{g} / \mathrm{ml})$ for 3 days. Viable cells (identified as being Annexin- $\mathrm{V}^{-} /$propidium iodide ${ }^{-}$) were FACS-isolated and injected subcutaneously into the axillae of female $\mathrm{Nu} / \mathrm{Nu}$ mice [5000 cells/axilla suspended in $100 \mu \mathrm{L}$ of DPBS and 200 $\mu \mathrm{L}$ of Matrigel (BD Biosciences)]. Tumor volume was assessed periodically using calipers, with volume calculated based on the modified ellipsoid formula $(\mathrm{L} \times \mathrm{W} \times \mathrm{W} / 2)$, where $\mathrm{L}$ represents length and $\mathrm{W}$ represents width. The mice were euthanized when tumors reached 1500 to $2000 \mathrm{~mm}^{3}$.

To examine the effect of Disulfiram in vivo, we first assessed the impact of Disulfiram on flank tumor growth. 100,000 bulk A2780 cells or 10,000 FACS-isolated CD133 ${ }^{+} / \mathrm{ALDH}^{+}$A2780 cells were injected into the flank of nude mice. Tumors were allowed to engraft for 1 week; then we initiated therapy with either vehicle, Disulfiram (0.15 mg i.p. daily for 21 days—dose equivalent of $500 \mathrm{mg} /$ day in humans [17]), Cisplatin (0.5 mg/kg daily for 3 days), or the combination of Cisplatin and Disulfiram either concurrently daily for 3 days, or concurrently daily for 3 days followed by an additional 18 days of Disulfiram therapy. Tumor volumes were measured using the LxWxW/2 formula for ellipsoid tumors. For IP experiments, 10,000 SKOV3 cell or 10,000 FACS-isolated $\mathrm{CD}_{133}{ }^{+} / \mathrm{ALDH}^{+} \mathrm{A} 2780$ cells were injected intraperitoneally into nude mice. Cells were allowed to engraft for 1 week then mice were treated daily for 21 days with Disulfiram ( $0.15 \mathrm{mg}$ i.p.). Controls received vehicle only. Mice were evaluated periodically for physical health and were sacrificed when fulfilling the UCUCA criteria of severe morbidity. Survival was estimated using the Kaplan-Meier plot.

\subsection{Flow Cytometric Analysis and Fluorescence-Activated Cell Sorting (FACS)}

A2780, OVSAHO, or HEY1 cells were harvested, counted in single-cell suspensions and plated (250,000 cells/well and 500,000 cells/well for the apoptosis induction and the ovarian CSC experiments, respectively) in $60 \mathrm{~mm}$ plates, then treated the following day with Disulfiram ( $7.5 \mu \mathrm{M}$ for 3 days and $15 \mu \mathrm{M}$ for 2 days for the apoptosis induction and the ovarian CSC experiments, respectively). Controls received vehicle only. Cells were harvested and counted; then FACS-sorted for the Annexin-V-positive cells (which indicates apoptosis induction) or CD133 positivity (which marks ovarian CSC).

In the apoptosis induction assays, cells were stained with Annexin-V and propidium iodide (PI), using the FITC Annexin V Apoptosis Detection Kit II (BD Biosciences) according to the manufacturer's instructions. This was followed by flow cytometric analysis.

In the assays examining the in vitro effect of Disulfiram on the ovarian CSC population using the CD133 antigen as a marker for stemness, A2780 cells were stained with anti-CD133/2-APC (MiltenyiBiotec, Auburn, CA), followed by ALDEFLUOR using the ALDEFLUOR assay (Stem Cell Technologies, Vancouver, BC, Canada). As a negative control, for each sample of stained cells an aliquot was treated with $50 \mathrm{mM}$ of diethylaminobenzaldehyde (DEAB), a specific ALDH inhibitor. This was followed by flow cytometric analysis. PI exclusion gates defined the viable cell population while the negative gates were established using the ALDEFLUOR/ 
DEAB treated cells as previously described [3].

In the in vivo experiments, where applicable, following FACS analysis, sterile sorting for the Annexin- $\mathrm{V}^{-} / \mathrm{PI}^{-}$ cells or the $\mathrm{CD}_{133^{+}} / \mathrm{ALDH}^{+}$cells was performed under low pressure in the absence of UV light.

For all experiments, flow cytometric analysis was performed by the University of Michigan Flow Cytometry Core using either the BD FACSAria or FACSDiva instruments (both from Becton Dickinson, San Diego, CA). FACS profiles were subsequently analyzed using FlowJo (Tree Star Inc., Ashland, OR).

\subsection{Statistical Analysis}

Mean values and standard deviations for all experiments were determined using Microsoft Excel software (Microsoft, Redmond, WA). Groups were compared using Student t-test (Microsoft Excel software). A p value of less than 0.05 was considered statistically significant. The $\mathrm{IC}_{50} \mathrm{~s}$ were estimated from the concentration/response curves. GraphPad Prism version 5.00 for Windows (GraphPad Inc., San Diego, CA) was used to generate Kaplan-Meier survival curves.

\section{Results}

We first examined the in vitro cytotoxicity of Disulfiram against bulk ovarian cancer cells. Using MTT assays, Disulfiram demonstrated an antineoplastic activity against a variety of ovarian cancer cell lines (Figure 1(a) \& Figure 1(b)), but was most effective in the platinum-sensitive (A2780, $\mathrm{IC}_{50}: \sim 1.5 \mu \mathrm{M}$ ) as opposed to the platinum-resistant (SKOV3, IC 50 : 40 $\mu \mathrm{M}$ ) cell lines. Consistent with previous results, we observed that Disulfiram induced apoptotic cell death (Supplemental Figure 1). We then compared the cytotoxicity of Disulfiram to that of Cisplatin, and found that the antineoplastic activity of the two drugs in the A2780 cell line is comparable (Cisplatin $\mathrm{IC}_{50}: \sim 3 \mu \mathrm{M}$, Figure 1(b)). We then examined, in vitro, the relative sensitivities of ovarian cancer cells or non-neoplastic human cells, including human ovarian surface epithelial cells (HOSE) and mesenchymal

(a)

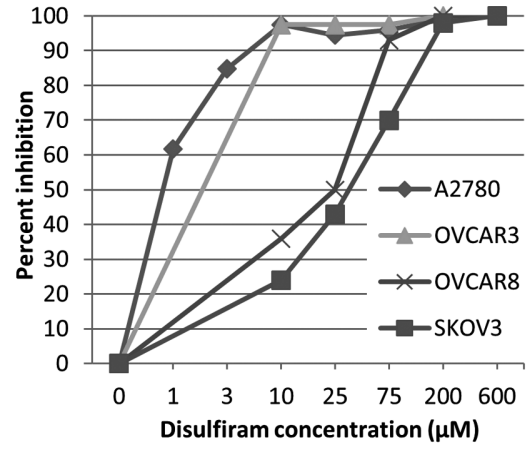

(c)

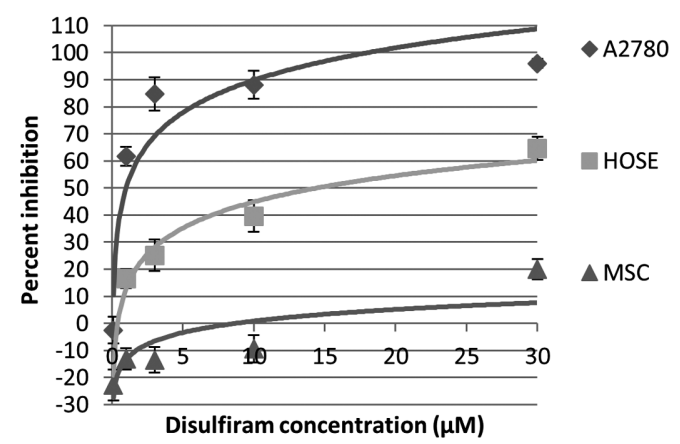

(b)

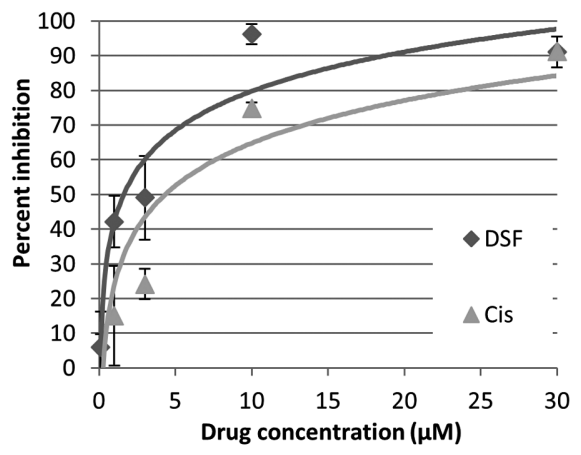

2780

(1)

Figure 1. In vitro cytotoxicity of Disulfiram against ovarian cancer: (a) MTT assays demonstrating percent inhibition of cell growth with increasing concentrations of Disulfiram (DSF) treatment versus untreated controls; (b) Comparison of percent inhibition of cell growth with Cisplatin (Cis) versus Disulfiram at various concentrations; (c) Comparison of growth inhibitory effects of Disulfiram on A2780 ovarian cancer cells (OCC) versus human ovarian surface epithelial cells (HOSE) versus mesenchymal stem cells (MSC) suggests that Disulfiram preferentially inhibits cancer cells. Results are representative of at least 3 assays with 3 replicates for each group. 
stem cells (MSC), to Disulfiram. We observed that ovarian cancer cells are most sensitive to Disulfiram. The HOSE cells were modestly sensitive to Disulfiram, while the MSC were relatively insensitive to Disulfiram (Figure 1(c)). This data suggests that ALDH inhibition with Disulfiram preferentially impact cancer cells.

As ALDH inhibition is reported to enhance chemotherapy response in numerous malignancies [10]-[14], we next tested the response of A2780 cells to Cisplatin, Disulfiram, or their combination. While Cisplatin alone had little activity, the combination of Disulfiram to Cisplatin increased activity (Figure 2(a)). As Cisplatin can increase the percentage of $\mathrm{ALDH}^{+}$cells [3], it is possible treating with cisplatin could increase resistance to Disulfiram. We therefore also examined the importance of drug sequence. Cells were treated with either drug alone, the two drugs given sequentially, or given concurrently. Treatment with concurrent Cisplatin and Disulfiram was superior to either drug as a single agent. Neither drug sequence (Cisplatin followed by Disulfiram or Disulfiram followed by Cisplatin) was better than single-agent therapy (Figure 2(b)).

Disulfiram has been reported to target CSC in breast cancer. We therefore next used flow cytometry to assess the ability of Disulfiram to specifically deplete marker-expressing ovarian CSC in vitro. As Disulfiram is an irreversible inhibitor of ALDH, we could not use ALDH expression as a measure of stemness. We therefore used the expression of CD133 and CD44 as an ovarian CSC marker to evaluate the CSC populationin different cell lines. At lower doses of Disulfiram, we observed clear induction of cell death but no selective death of CD133 cells (Figure 3 and data not shown). At higher concentrations of Disulfiram we saw modest ( 1.2 fold) preferential depletion of the CD133 ${ }^{+}$cellsbut up to 1.9-4-fold depletion of CD44 ${ }^{+}$cells (Figure 3).

We then tested the impact of Disulfiram on the growth of ovarian tumor spheres, which is a functional assay for tumor CSC growth. Compared to mock-treated cells, cells treated with Disulfiram showed a concentrationdependent reduction in tumor sphere formation. This was demonstrated in both cell lines and primary patient samples (Figure 4(a) and Figure 4(b)).

We next assessed the ability of Disulfiram to impact the tumor initiation capacity of ovarian cancer cells. We treated the A2780 cell line in vitro with Disulfiram, Cisplatin, or vehicle and FACS-isolated viable (Annexin- $\left.\mathrm{V}^{-} / \mathrm{PI}^{-}\right)$cells. 5000 viable cells from each group were then injected into immunodeficient mice. We observed

(a)

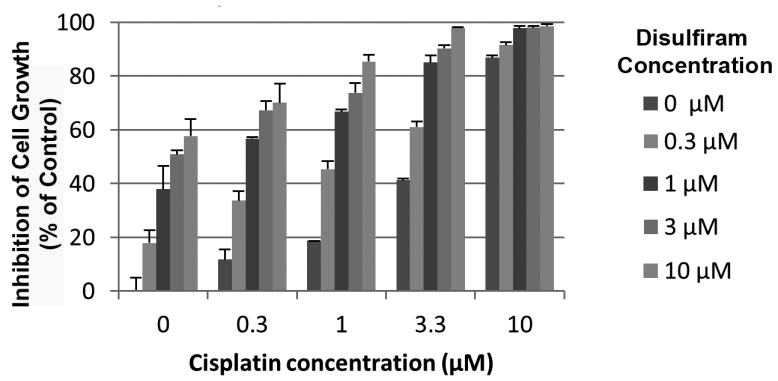

(b)

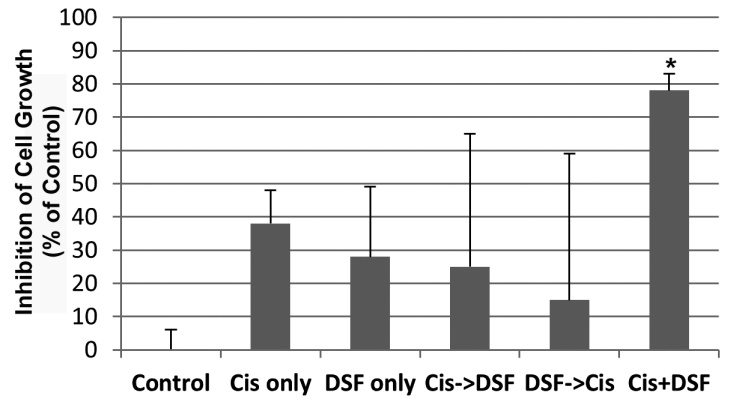

Figure 2. Disulfiram increases the cytotoxicity of Cisplatin. (a) Percent inhibition of A2780 cell growth with increasing doses of Cisplatin combined with increasing doses of Disulfiram (DSF); (b) Drug sequencing study demonstrating that concurrent Cisplatin (Cis) and Disulfiram (Cis + DSF) is superior to Cisplatin therapy followed by Disulfiram therapy (Cis- $>$ DSF), Disulfiram therapy followed by Cisplatin (DSF- > Cis), or either drug as a single agent. Results are representative of at least 2 assays with 3 replicates for each group. *indicates $\mathrm{p}<0.05$. 
A2780

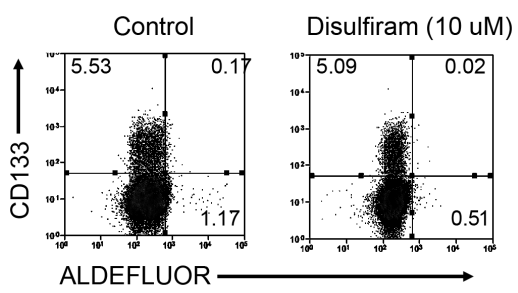

ALDEFLUOR

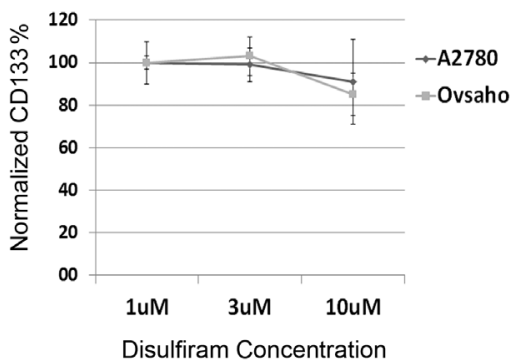

Hey1

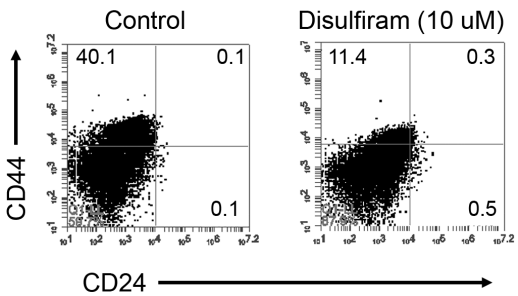

(a)

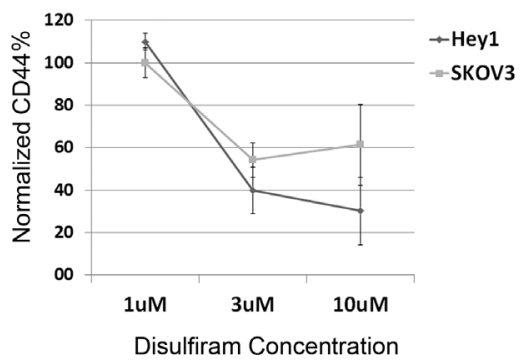

(b)

Figure 3. Disulfiram's impact on ovarian CSC marker expressionin vitro. (a) CD133 FACS analysis of A2780 and HEY1 cells treated with $10 \mu \mathrm{M}$ Disulfiram demonstrating a minimal depletion of the $\mathrm{CD} 133^{+}$ovarian cancer cellpopulation and 3.9 fold reduction of the CD44 ${ }^{+}$cell population with Disulfiram treatment; (b) Dose dependent effect of Disulfiram on $\mathrm{CD}_{133^{+}}$cell and CD44 ${ }^{+}$ovarian cancer cell populations for the indicated cell lines. The percentage of cells expressing CD133 or CD44 at each treatment dose are presented as normalized to the percent of marker expressing cells in the untreated control.

SKOV3
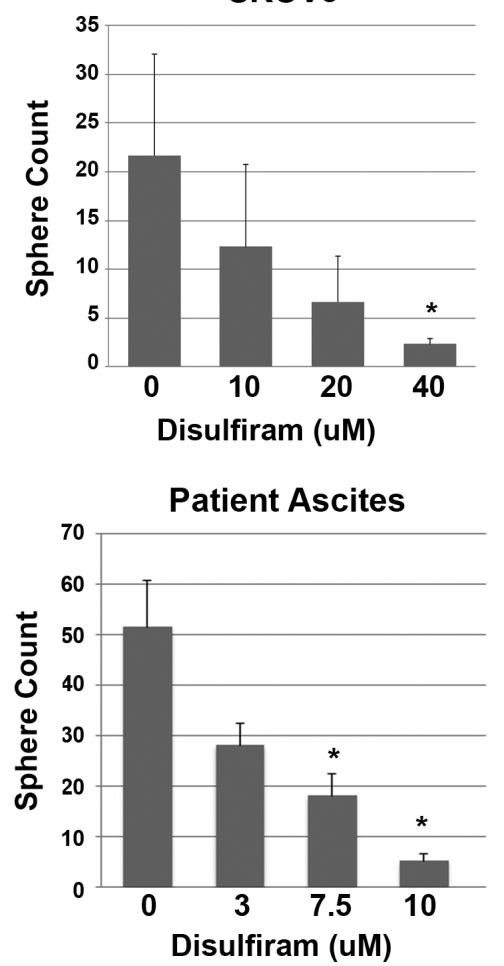

A2780

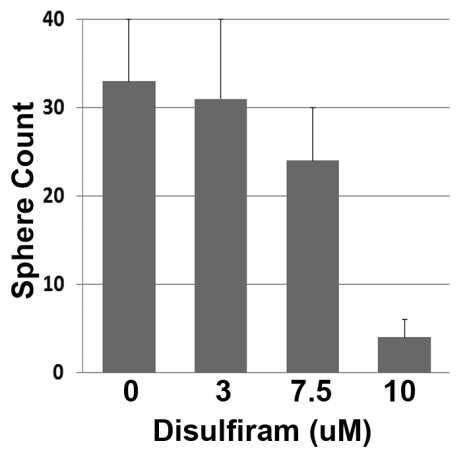

Primary ALDH+CD133+

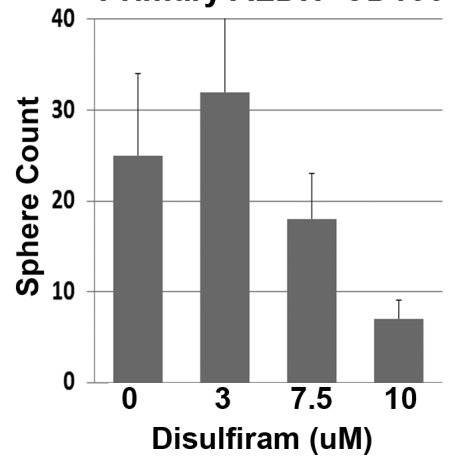

(a) 

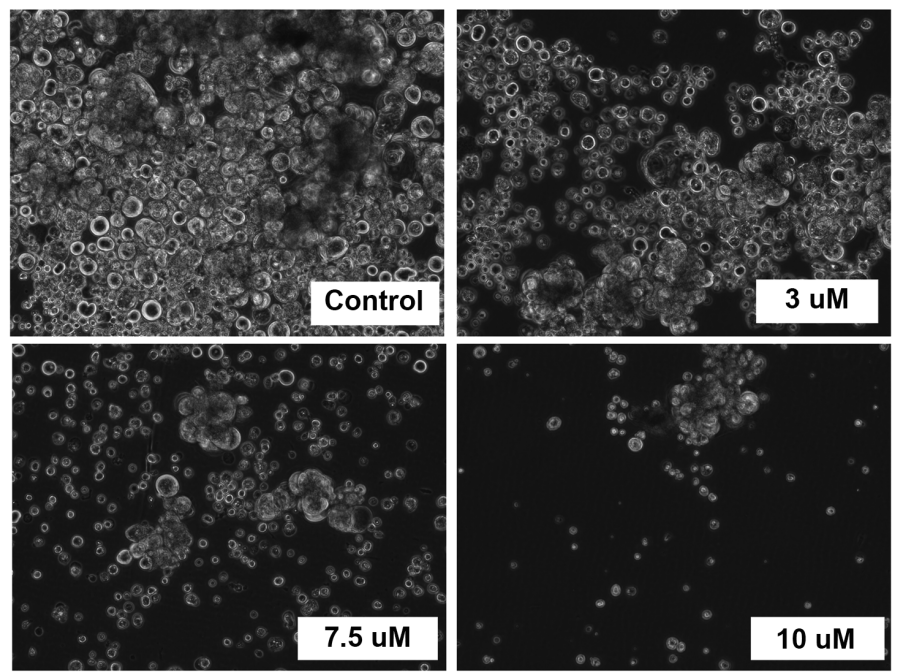

(b)

\begin{abstract}
Figure 4. Disulfiram's impact on tumor sphere formation. (a) Tumor sphere count from A2780 and SKOV3 cell lines or FACS isolated primary $\mathrm{ALDH}^{+} \mathrm{CD}_{133^{+}}$ primary cells or primary patient ascites samples $(n=3)$ treated with the indicated concentrations of Disulfiram (DSF) for 3 days, then maintained for 14 and 20 days respectively; (b) Representative pictures of primary patient ascites samples at the end of the culture period. Results are representative of at least 2 assays with 3 replicates for each group. *indicates $\mathrm{p}<0.05$.
\end{abstract}

no impact on tumor initiation or growth with either Cisplatin or Disulfiram (Figure 5(a)).

We then examined the effect of Disulfiram on flanks tumors derived from either whole A2780 cell line or from FACS-isolated $\mathrm{ALDH}^{+} / \mathrm{CD}_{133^{+}}$A2780 CSC. Disulfiram alone had no statistically significant impact on the growth of either tumor population. Similarly the addition of Disulfiram to a sub-therapeutic dose of Cisplatin (concurrently or concurrently with consolidative Disulfiram) had no impact on tumor growth (Figure 5(b)). In order to increase drug exposure to tumors we repeated a therapeutic study using intraperitoneal treatment of FACS-isolated ALDH ${ }^{+} / \mathrm{CD}_{13}{ }^{+}$A2780 cells injected intraperitoneally into NSG mice. Xenografts were allowed to establish for 1 week prior to initiation of treatment. Mice were then treated with Disulfiram or vehicle i.p. for 21 days. Survival curves showed no impact on survival with Disulfiram treatment (Figure 5(c)). Finally, given the increased CD44 depletion in vitro with Disulfiram vs. SKOV and HEY1 cells, we established IP SKOV tumors and repeated dosing. Once again no overall survival effect was observed (Figure 5(c)).

\title{
4. Discussion
}

Disulfiram, as an inhibitor of ALDH enzymatic activity, has been reported to selectively target CSC. Given its relatively low cost, ease of administration, and acceptable toxicity profile, Disulfiram would be an attractive CSC-targeting therapeutic agent. Agents that target CSC are reported to overcome chemotherapy resistance and increase survival in murine tumor models. Given studies from our lab and others linking ALDH activity in ovarian cancer cells with increased tumor initiation capacity and chemotherapy resistance, we tested the ability of Disulfiram to act as a CSC-targeting agent in ovarian cancer. We found that Disulfiram demonstrated significant anti-tumor activity in vitro, which was additive to chemotherapy. However, while Disulfiram effectively targeted $\mathrm{CD} 44^{+}$ovarian cancer cells, Disulfiram demonstrated little selective elimination of $\mathrm{ALDH}^{+}$or $\mathrm{CD} 133^{+}$ ovarian cancer-initiating cells in vitro and lacked efficacy against CSC in vivo.

Our results differ from prior studies in breast cancer, in which Disulfiram was reported to target breast cancer stem-like cells [11]. Some studies have used copper to enhance the effects of Disulfiram [9]-[11] [14]. We did not use copper, and this may have reduced efficacy. Similarly, the dosing we used in this study is less than that used in other studies and may impact the lack of efficacy. We chose to use a dose reflective of a $500 \mathrm{mg}$ daily maintenance dose used in humans for the treatment of alcohol addiction. We hypothesize a maintenance dose could prevent or delay disease recurrence. We found higher doses were limited by neurologic side-effects in the 

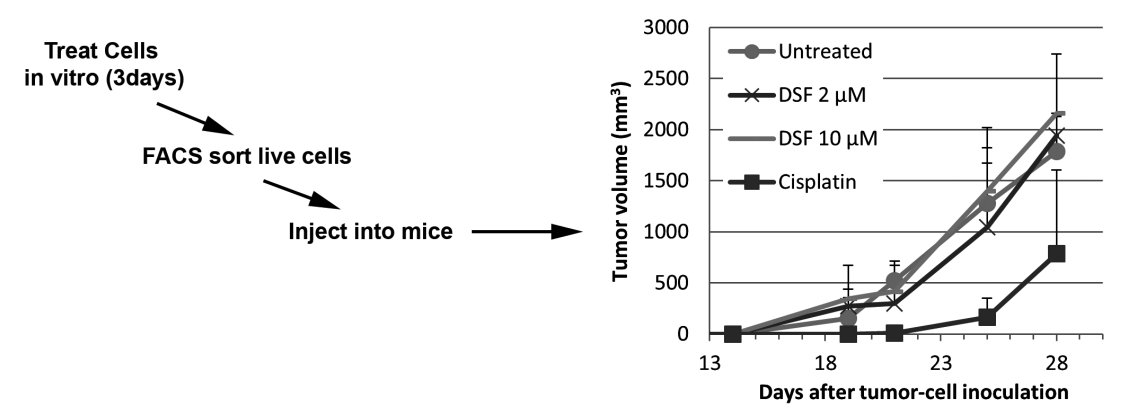

(a)
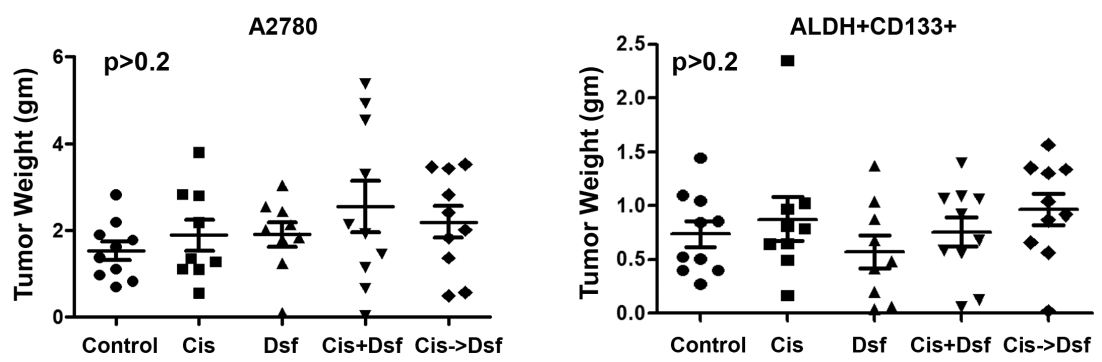

(b)
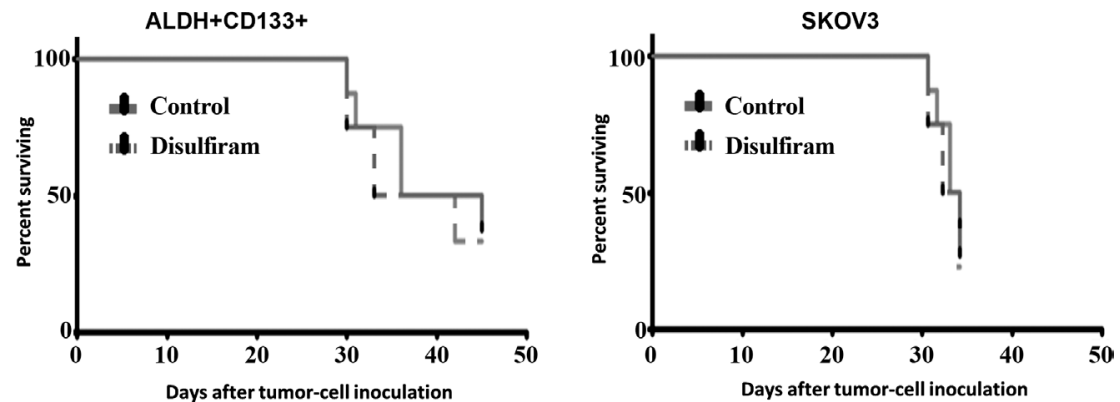

(c)

Figure 5. Disulfiram does not impact tumor initiation or cancer stem-like cells (CSC) growth in vivo. (a) A2780 cells were mock-treated or treated with Cisplatin (Cis) or Disulfiram (DSF) for 3 days. 5000 FACS-isolated viable (Annexin-V/PI') cells from each group were injected into the axillae of nude mice and tumor initiation and growth were tracked. Disulfiram had no impact on tumor initiation or growth; (b) Nude mice were injected in the flank with bulk A2780 cells or FACS-isolated $\mathrm{ALDH}^{+} / \mathrm{CD} 133^{+}$ A2780 cells, then treated with vehicle, Cisplatin (Cis, $0.25 \mathrm{mg} / \mathrm{kg}$ i.p. daily for 3 days), Disulfiram (DSF, 0.15 mg i.p. daily for 21 days), concurrent Cisplatin and Disulfiram for 3 days (Cis + DSF), or Cisplatin for 3 days followed by Disulfiram for 18 days (Cis- > DSF). Disulfiram had no impact on tumor growth; (c) Nude mice were injected i.p. with 10,000 FACS-isolated CD133 ${ }^{+} / \mathrm{ALDH}^{+}$A2780 cells, or total SKOV3 cells and then treated daily for 21 days with i.p. Disulfiram or vehicle. Mice were evaluated periodically and were sacrificed when fulfilling University Committee on Use and Care of Animals (UCUCA) criteria of severe morbidity. Kaplan-Meier survival curves showed no impact on survival with Disulfiram treatment in either case.

mice. While higher doses have been used in humans, up to 2 grams/day, this is associated with much higher side-effects and would not be acceptable as a maintenance approach. A likely explanation for the different activity of Disulfiram in vitro versus in vivo is reduced bioavailability. Disulfiram has a very short half-life in vivo and numerous active metabolites [18]-[21], and these multiple metabolites of Disulfiram are associated with numerous off-target effects [9]-[11]. The tumor microenvironment may further restrict bioavailability. In an effort to maximize in vivo exposure, we used an intraperitoneal tumor model and intraperitoneal drug administration. Despite this, we still saw limited in vivo Disulfiram effect. 
The reason for the differential effect of Disulfiram in vitro vs. in vivo remains unclear. One reason that Disulfiram might have been unsuccessful is that it is inadequately/not targeting the ALDH isozymes selectively expressed in CSC. There are at least 19 ALDH isozymes in the human aldehyde dehydrogenase superfamily [22]. The ALDEFLUOR assay that is used to identify CSC detects the enzymatic activity of multiple ALDH isozymes [23]. The exact isozymes that are linked with ovarian CSC ALDH activity remain unknown. Most studies have implicated ALDH1A1 as a marker of ovarian CSC; however, other ALDH1A family members and ALDH3 family members have also been implicated [24] [25]. Disulfiram was developed clinically based on its high-affinity inhibition of ALDH2 [26]. ALDH2 is a broadly expressed mitochondrial enzyme and, as such, is likely not a CSC-specific target. Although Disulfiram can inhibit ALDH1 in vitro [27], the in vivo activity of the drug is mainly attributed to its metabolites targeting mitochondrial ALDH2 [18]-[21]. Thus, Disulfiram may result in limited inhibition of ALDH1A1 in vivo.

\section{Conclusion}

In conclusion, ALDH inhibition with Disulfiram shows anti-neoplastic activity in vitro. However, in vivo activity was limited. Our results suggest that Disulfiram is not the ideal ALDH inhibitor for ovarian cancer and that more selective may be necessary to efficiently target CSC. A more comprehensive examination of the Disulfiram, its pharmacokinetics and combination with chemotherapy in vivo are indicated prior to use in clinical trials in ovarian cancer patients.

\section{References}

[1] Boman, B.M. and Wicha, M.S. (2008) Cancer Stem Cells: A Step toward the Cure. Journal of Clinical Oncology, 26, 2795-2799. http://dx.doi.org/10.1200/JCO.2008.17.7436

[2] Ginestier, C., Hur, M.H., Charafe-Jauffret, E., Monville, F., Dutcher, J., Brown, M., et al. (2007) ALDH1 Is a Marker of Normal and Malignant Human Mammary Stem Cells and a Predictor of Poor Clinical Outcome. Cell Stem Cell, 1, 555-567. http://dx.doi.org/10.1016/j.stem.2007.08.014

[3] Silva, I.A., Bai, S., McLean, K., Yang, K., Griffith, K., Thomas, D., et al. (2011) Aldehyde Dehydrogenase in Combination with CD133 Defines Angiogenic Ovarian Cancer Stem Cells That Portend Poor Patient Survival. Cancer Research, 71, 3991-4001. http://dx.doi.org/10.1158/0008-5472.CAN-10-3175

[4] Clarke, M.F., Dick, J.E., Dirks, P.B., Eaves, C.J., Jamieson, C.H., Jones, D.L., et al. (2006) Cancer Stem Cells-Perspectives on Current Status and Future Directions: AACR Workshop on Cancer Stem Cells. Cancer Research, 66, 9339-9344. http://dx.doi.org/10.1158/0008-5472.CAN-06-3126

[5] Choi, Y., Ingram, P.N., Yang, K., Coffman, Y., Iyengar, M., Bai, S., et al. (2015) Identifying an Ovarian Cancer Hierarchy Regulated by BMP2. PNAS, In Press. http://dx.doi.org/10.1073/pnas.1507899112

[6] Landen, C.N., Goodman, B., Katre, A.A., Steg, A.D., Nick, A.M., Stone, R.L., et al. (2010) Targeting Aldehyde Dehydrogenase Cancer Stem Cells in Ovarian Cancer. Molecular Cancer Therapeutics, 9, 3186-3199. http://dx.doi.org/10.1158/1535-7163.MCT-10-0563

[7] Steg, A.D., Bevis, K.S., Katre, A.A., Ziebarth, A., Dobbin, Z.C., Alvarez, R.D., et al. (2012) Stem Cell Pathways Contribute to Clinical Chemoresistance in Ovarian Cancer. Clinical Cancer Research, 18, 869-881. http://dx.doi.org/10.1158/1078-0432.CCR-11-2188

[8] Lindahl, R. (1992) Aldehyde Dehydrogenases and Their Role in Carcinogenesis. Critical Reviews in Biochemistry and Molecular Biology, 27, 283-335. http://dx.doi.org/10.3109/10409239209082565

[9] Chen, D., Cui, Q.C., Yang, H. and Dou, Q.P. (2006) Disulfiram, a Clinically Used Anti-Alcoholism Drug and Copper-Binding Agent, Induces Apoptotic Cell Death in Breast Cancer Cultures and Xenografts via Inhibition of the Proteasome Activity. Cancer Research, 66, 10425-10433. http://dx.doi.org/10.1158/0008-5472.CAN-06-2126

[10] Zhang, H., Chen, D., Ringler, J., Chen, W., Cui, Q.C., Ethier, S.P., et al. (2010) Disulfiram Treatment Facilitates Phosphoinositide 3-Kinase Inhibition in Human Breast Cancer Cells in Vitro and in Vivo. Cancer Research, 70, 3996-4004. http://dx.doi.org/10.1158/0008-5472.CAN-09-3752

[11] Yip, N.C., Fombon, I.S., Liu, P., Brown, S., Kannappan, V., Armesilla, A.L., et al. (2011) Disulfiram Modulated ROS-MAPK and NF- $\kappa$ B Pathways and Targeted Breast Cancer Cells with Cancer Stem Cell-Like Properties. British Journal of Cancer, 104, 1564-1574. http://dx.doi.org/10.1038/bjc.2011.126

[12] Wang, W., McLeod, H.L. and Cassidy, J. (2003) Disulfiram-Mediated Inhibition of NF- $\kappa$ B Activity Enhances Cytotoxicity of 5-Fluorouracil in Human Colorectal Cancer Cell Lines. International Journal of Cancer, 104, 504-511. http://dx.doi.org/10.1002/ijc.10972 
[13] Lin, J., Haffner, M.C., Zhang, Y., Lee, B.H., Brennen, W.N., Britton, J., et al. (2011) Disulfiram Is a DNA Demethylating Agent and Inhibits Prostate Cancer Cell Growth. Prostate, 71, 333-343. http://dx.doi.org/10.1002/pros.21247

[14] Morrison, B.W., Doudican, N.A., Patel, K.R. and Orlow, S.J. (2010) Disulfiram Induces Copper-Dependent Stimulation of Reactive Oxygen Species and Activation of the Extrinsic Apoptotic Pathway in Melanoma. Melanoma Research, 20, 11-20. http://dx.doi.org/10.1097/CMR.0b013e328334131d

[15] Raha, D., Wilson, T.R., Peng, J., Peterson, D., Yue, P., Evangelista, M., et al. (2014) The Cancer Stem Cell Marker Aldehyde Dehydrogenase Is Required to Maintain a Drug-Tolerant Tumor Cell Subpopulation. Cancer Research, 74, 3579-3590. http://dx.doi.org/10.1158/0008-5472.CAN-13-3456

[16] Coffman, L., Mooney, C., Lim, J., Bai, S., Silva, I., Gong, Y., et al. (2013) Endothelin Receptor-A Is Required for the Recruitment of Antitumor T Cells and Modulates Chemotherapy Induction of Cancer Stem Cells. Cancer Biology \& Therapy, 14, 184-192. http://dx.doi.org/10.4161/cbt.22959

[17] Vaccari, A., Ferraro, L., Saba, P., Ruiu, S., Mocci, I., Antonelli, T., et al. (1998) Differential Mechanisms in the Effects of Disulfiram and Diethyldithiocarbamate Intoxication on Striatal Release and Vesicular Transport of Glutamate. Journal of Pharmacology and Experimental Therapeutics, 285, 961-967.

[18] Yourick, J.J. and Faiman, M.D. (1991) Disulfiram Metabolism as a Requirement for the Inhibition of Rat Liver Mitochondrial Low Km Aldehyde Dehydrogenase. Biochemical Pharmacology, 42, 1361-1366.

http://dx.doi.org/10.1016/0006-2952(91)90446-C

[19] Hart, B.W. and Faiman, M.D. (1992) In Vitro and in Vivo Inhibition of Rat Liver Aldehyde Dehydrogenase by S-Methyl N, N-Diethylthiolcarbamate Sulfoxide, a New Metabolite of Disulfiram. Biochemical Pharmacology, 43, 403-406. http://dx.doi.org/10.1016/0006-2952(92)90555-W

[20] Madan, A. and Faiman, M.D. (1994) Diethyldithiocarbamate Methyl Ester Sulfoxide, an Inhibitor of Rat Liver Mitochondrial Low Km Aldehyde Dehydrogenase and Putative Metabolite of Disulfiram. Alcoholism: Clinical and Experimental Research, 18, 1013-1017. http://dx.doi.org/10.1111/j.1530-0277.1994.tb00075.x

[21] Lipsky, J.J., Shen, M.L. and Naylor, S. (2001) In Vivo Inhibition of Aldehyde Dehydrogenase by Disulfiram. ChemicoBiological Interactions, 130-132, 93-102. http://dx.doi.org/10.1016/S0009-2797(00)00225-8

[22] Vasiliou, V. and Nebert, D.W. (2005) Analysis and Update of the Human Aldehyde Dehydrogenase (ALDH) Gene Family. Human Genomics, 2, 138-143.

[23] Moreb, J.S., Ucar, D., Han, S., Amory, J.K., Goldstein, A.S., Ostmark, B., et al. (2012) The Enzymatic Activity of Human Aldehyde Dehydrogenases 1A2 and 2 (ALDH1A2 and ALDH2) Is Detected by Aldefluor, Inhibited by Diethylaminobenzaldehyde and Has Significant Effects on Cell Proliferation and Drug Resistance. Chemico-Biological Interactions, 195, 52-60. http://dx.doi.org/10.1016/j.cbi.2011.10.007

[24] Marchitti, S.A., Orlicky, D.J., Brocker, C. and Vasiliou, V. (2010) Aldehyde Dehydrogenase 3B1 (ALDH3B1): Immunohistochemical Tissue Distribution and Cellular-Specific Localization in Normal and Cancerous Human Tissues. Journal of Histochemistry and Cytochemistry, 58, 765-783. http://dx.doi.org/10.1369/jhc.2010.955773

[25] Rosen, J.M. and Jordan, C.T. (2009) The Increasing Complexity of the Cancer Stem Cell Paradigm. Science, 324, 1670-1673. http://dx.doi.org/10.1126/science.1171837

[26] Sládek, N.E. (2003) Human Aldehyde Dehydrogenases: Potential Pathological, Pharmacological, and Toxicological Impact. Journal of Biochemical and Molecular Toxicology, 17, 7-23. http://dx.doi.org/10.1002/jbt.10057

[27] Moore, S.A., Baker, H.M., Blythe, T.J., Kitson, K.E., Kitson, T.M. and Baker, E.N. (1998) Sheep Liver Cytosolic Aldehyde Dehydrogenase: The Structure Reveals the Basis for the Retinal Specificity of Class 1 Aldehyde Dehydrogenases. Structure, 6, 1541-1551. http://dx.doi.org/10.1016/S0969-2126(98)00152-X 